\title{
Small Angle X-Ray Scattering: Getting the Big Picture of the Very Small
}

Pierre Panine, Manuel Fernandez-Martinez, Sergio Rodrigues, Ronan Mahe, Frédéric Bossan and Peter Høghøj

Xenocs SA, Sassenage, France

Small Angle X-ray Scattering (SAXS) is a technique for characterizing the nano-structure of materials [1]. By recording the x-ray scattering pattern, one obtains reciprocal space information that can be transformed or modeled to extract real-space information from the sample (see Fig. 1 for an example).

The information obtained in one measurement is statistically representative of the volume probed by the $\mathrm{x}$-ray beam, which typically is about $1 \mathrm{~mm}^{3}$. The technique is non-destructive for the sample and as with X-ray CT and imaging, structural information can be obtained from inside a sample with little sample preparation. These features make the technique a valuable tool and a complement to microscopy and real-space imaging methods.

The short wavelength used allows for obtaining structural information in the range from $1 \mathrm{~nm}$ to beyond $100 \mathrm{~nm}$. By combining SAXS with Wide Angle X-Ray Scattering (WAXS), the lower limit can be extended to below $0.1 \mathrm{~nm}$ and include information on crystalline structure and phase.

A broad range of materials and applications can be addressed by SAXS and WAXS techniques, ranging from soft condensed matter, polymers, bio-materials to nanoparticles, fibers and even structured surfaces which can be measured in the Grazing Incidence (GISAXS) geometry.

The capability of SAXS to measure the size of nano-particles is recognized in the ISO standard currently being drafted [2] and the technique is also well suited for measuring size distributions or average shape and also surface to volume ratios.

In recent years there has been a strong interest in the SAXS technique, partly because it addresses a need for characterization related to nanotechnology and nano-materials, but also because there has been considerable progress in both synchrotron and also laboratory instrumentation. Faster measurements and better data has also opened the way towards dynamic measurements with parameters such as temperature, humidity, pressure, DSC, flow and also the use of the technique for quality assurance.

We will present SAXS results from a broad range of samples with the aim to discuss how this type of data can be a complement to microscopy and other analytical methods.

[1] Glatter O, Kratky O, ed. (1982). Small Angle X-ray Scattering. Academic Press., (downloadable from http://physchem.kfunigraz.ac.at/sm/Software.htm )

[2] ISO/NP 17867 currently in preparation by committee ISO/TC24/SC4/WG10 


\section{Biological solution (microtubulin) $4.24 \mathrm{mg} / \mathrm{ml}$}
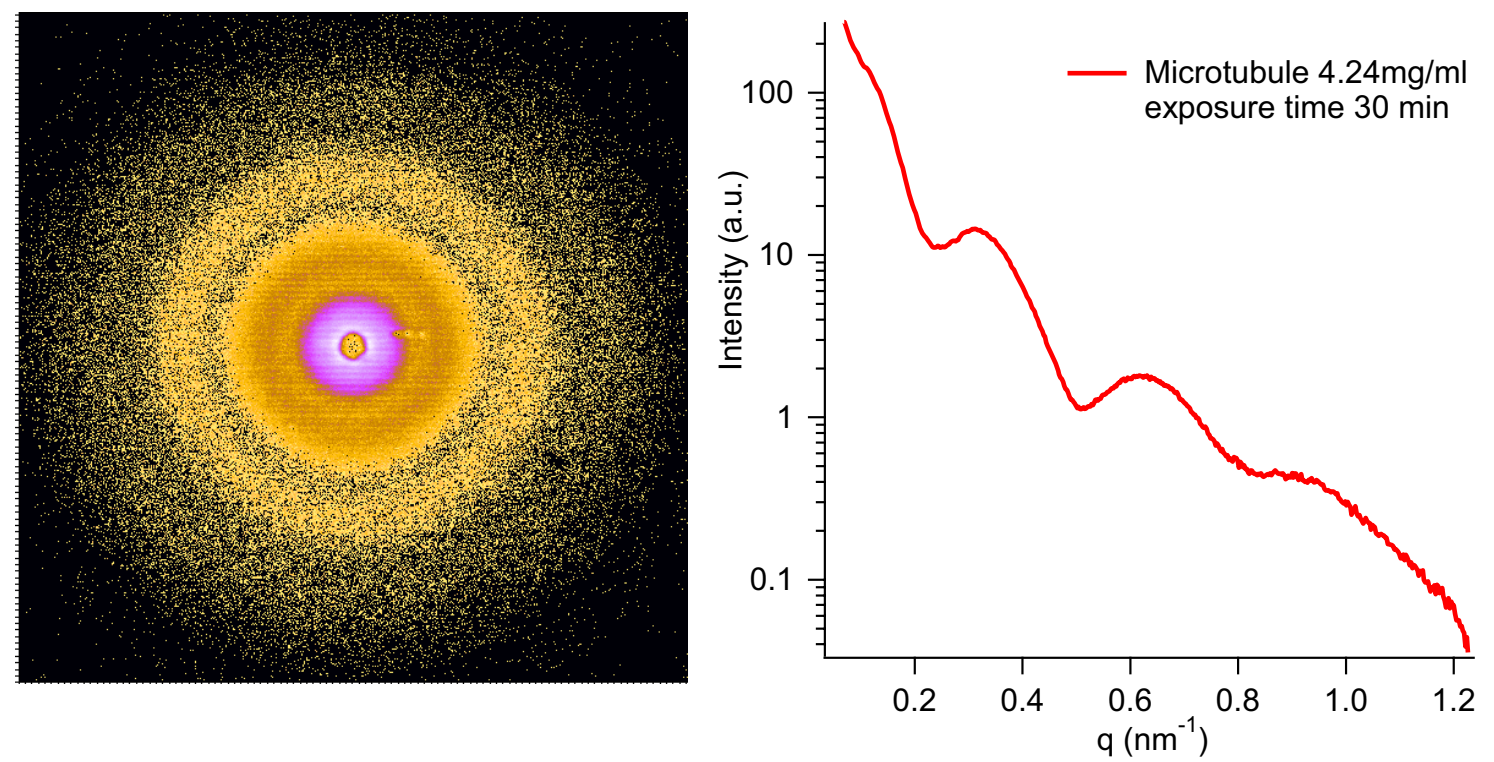

Figure 1. SAXS pattern from solution of microtubulin $(4.24 \mathrm{mg} / \mathrm{ml})$. After azimuthal integration, intensity oscillations are clearly visible with a periodicity of app. $0.3 \mathrm{~nm}^{-1}$, corresponding to a dimension of app. $21 \mathrm{~nm}$ 